\title{
Cretaceous and Cenozoic decapod crustaceans of Jamaica
}

\author{
Stephen K. Donovan ${ }^{1}$, Roger W. Portell ${ }^{2}$ \& Joe S.H. Collins ${ }^{3}$ \\ ${ }^{1}$ Department of Palaeontology, Nationaal Natuurhistorisch Museum, Postbus 9517, NL-2300 RA Leiden, The \\ Netherlands; ${ }^{2}$ Florida Museum of Natural History, P.O. Box 117800, University of Florida, Gainesville, \\ Florida 32611-7800, USA; ${ }^{3}$ Department of Palaeontology, The Natural History Museum, Cromwell Road, \\ London SW7 5BD, England, and 8, Shaw's Cottages, Perry Rise, Forest Hill, London SE23 2QN, England
}

Keywords: Jamaica, Cretaceous, Cenozoic, Crustacea, Decapoda

\begin{abstract}
In the last decade, a rebirth in interest of Jamaican fossil crustaceans has occurred. A summary of known material is provided together with some indications of the directions that future studies should take.
\end{abstract}

\section{Introduction}

The past ten years have seen a renaissance in the study of the fossil shrimps and crabs of Jamaica. Previously, the only publications on the island's decapod crustaceans were a trio of papers by Withers $(1922,1924,1927)$, which described taxa from the Upper Cretaceous and Eocene. After this flurry of activity in the $1920 \mathrm{~s}$, Jamaican fossil shrimps and crabs were largely ignored until the late 1980 s, when Ms Carla Gordon of the University of the West Indies (UWI) collected abundant fragmentary material from the Falmouth Formation (Late Pleistocene, last interglacial). This material formed a significant part of the new collections reported on by Morris (1993) in the first paper on Jamaican crabs in 66 years. Since then the authors, aided by Mr H.L. Dixon (formerly UWI) and other collectors, have been describing the kocally abundant decapod remains found in Miocene and younger deposits of the island (Collins et al., 1997, 2001; Collins \& Donovan, 1998; Collins \& Portell, 1998; Donovan \& Dixon, 1998; Portell \& Collins, in press). Our current knowledge of Jamaican fossil shrimps and crabs is summarized in Table 1 , to which should be added the indeterminate specimens listed in
Morris (1993) and new, but as yet undescribed, collections mentioned below.

\section{Overview of material}

The range of depositional settings shown by the decapod-bearing units studied by the authors precludes any suggestion of a recurrent mode of preservation. Complete carapaces are rare, except in the Lower Miocene Montpelier Formation, where a diverse fauna has been collected within slide blocks of scleractinian corals. Although derived from a shallow-water setting, they are preserved in deepwater chalks (Portell \& Collins, in press), with a depositional depth in excess of $200 \mathrm{~m}$ (Underwood \& Mitchell, in press). Of particular interest here is the presence of the symbiotic genus Trapezia Latreille, a genus commensal with pocilloporid scleractinians, a group of corals no longer present in the Caribbean (with the exception of Madracis). Other deposits include the Bowden shell bed (submarine mass flow, laid down in 100-200 $\mathrm{m}$ water depth; Pickerill et al., 1998) of Late Pliocene age, the raised reef of the Late Pleistocene Falmouth Formation (Larson, 1983), coeval shallow-water siliciclastic lagoonal deposits of the Port Morant Formation (Mitchell et al., 2001) and a land crab claw in a fissure fill of terra rosa (Donovan \& Dixon, 1998).

That the early studies of Withers gave an incorrect impression of the true stratigraphic and taxonomic diversity of Jamaican decapod crustaceans was determined by the research interests of the 
Table 1. Fossil shrimps and crabs from the Cretaceous and Cenozoic of Jamaícax Morris (1993) listed localities and horizons that have yielded indeterminate crab specimens. Data from Morris (1993) unless stated otherwise. Higher classification follows Martin \& Davis (2001). Key; $+=$ present; cf, = confer; aff. = affinity (see Bengtson, 1988, for a discussion of cf, and aff, in open nomenclature); $1=$ Upper Cretaceous (horizon unknown); 2 = "Veniella Shales" (Campanian); 3 = Guinea Corn Formation (Campanian-Maastrichtian); 4 = Chapelton Formation, Yellow Limestone Group (Early-Middle Eocene); 5 = White Limestone Group formation unknown (EoceneOligocene); 6= Montpelier Formation, White Límestone Group (Early Miocene) (Portell \& Collins, in press); $7=$ Bowden shell bed, Bowden Formation, Lower Coastal Group (Late Pliocene) (Collins \& Portell, 1998); $8=$ Old Pera beds, Manchioneal Formation, Upper Coastal Group (Early Pleistocene) (Collins et al,, 2001); $9=$ Falmouth Formation, Upper Coastal Group (Late Pleistocene); 10 = Port Morant Formation, Upper Coastal Group (Late Pleistocene) (Collins et al., 1997; Collins \& Donovan, 1998); 11 = fissure fill (Quaternary) (Donovan \& Dixon, 1998). For a general discussion of the geology of Jamaica, see Robinson (1994).

\begin{tabular}{|c|c|c|c|c|c|c|c|c|c|c|c|}
\hline Taxon & 1 & 2 & 3 & 4 & 5 & 6 & 7 & 8 & 9 & 10 & 11 \\
\hline \multicolumn{12}{|l|}{ Infraorder Thalassinidea } \\
\hline \multicolumn{12}{|l|}{ Family Callianassidae } \\
\hline "Callianassa" spp. & & & & + & + & & + & & + & & \\
\hline 'Callianassa' gigantea Withers & & & & + & & & & & & & \\
\hline 'Callianassa' subplana Withers & & & & + & & & & & & & \\
\hline 'Callianassa' trechmanni Withers & & & & + & & & & & & & \\
\hline Neocallichirus peraensis Collins et al. & & & & & & & & & & + & \\
\hline Glypturus acanthochirus Stimpson & & & & & & & & & & + & \\
\hline Family Ctenochelidae & & & - & & & & & & & & \\
\hline Ctenocheles sp. & & & & & & & + & & & & \\
\hline \multicolumn{12}{|l|}{ Infraorder Anomura } \\
\hline \multicolumn{12}{|l|}{ Family Porcellanidae } \\
\hline Petrolisthes sp. & & & & & & & & & + & & \\
\hline \multicolumn{12}{|l|}{ Family Albuneidae } \\
\hline Albunea sp. & & & & & & & & & + & & \\
\hline \multicolumn{12}{|l|}{ Family Diogenidae } \\
\hline Paguristes sp. & & & & & & & + & & & & \\
\hline Petrochirus sp. & & & & & & & & & + & & \\
\hline Petrochirus bahamensis (Herbst) & & & & & & & ef. & & & + & \\
\hline \multicolumn{12}{|l|}{ Infraorder Brachyura } \\
\hline \multicolumn{12}{|l|}{ Family Dromídae } \\
\hline Kromtitis sp. nov. & & & & & & + & & & & & \\
\hline \multicolumn{12}{|l|}{ Family Dynomenidae } \\
\hline Dynomene sp. nov. & & & & & & + & & & & & \\
\hline \multicolumn{12}{|l|}{ Family Raninidae } \\
\hline Cretacoranina trechmanni (Withers) & & + & & & & & & & & & \\
\hline Raninoides louisianensis Rathbun & & & & & & & & & & + & \\
\hline \multicolumn{12}{|l|}{ Family Calappidae } \\
\hline Calappa gallus (Herbst) & & & 1 & & & & & & cf: & & \\
\hline Calappa springeri Rathbun & & & $t$ & & & & aff. & & & + & \\
\hline \multicolumn{12}{|l|}{ Family Hepatidae } \\
\hline Eriosachila bartholomaeensis (Rathbun) & & & & + & & & & & & & \\
\hline Hepatus sp. & & & & & & & + & & & & \\
\hline Hepatus praecox Collins et al. & & & & & & & & * & & + & \\
\hline \multicolumn{12}{|l|}{ Family Necrocarcinidae } \\
\hline Necrocarcinus sp. & + & & & & & & & & & & \\
\hline Paranecrocarcinus? sp. & + & & & & & & & & & & \\
\hline Family Leucosiidae & & & 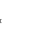 & & & & & & & & \\
\hline leucosild gen. et sp. nov. & & & & & & + & & & & & \\
\hline Persephona punctata punctata (Linné) & & & & & & & aff. & & & + & \\
\hline Uhlias limbatus Stimpson & & & & & & & & & cf. & & \\
\hline \multicolumn{12}{|l|}{ Family Mithracidae } \\
\hline Mithraculus forceps A. Milne Edwards & & & & & & & & & cf. & & \\
\hline Mithrax sp. & & & & & & & + & & & + & 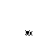 \\
\hline
\end{tabular}


Table 1. Continued.

Taxon
Mithrax sp. nov. A
Mithrax sp. nov. B
Mithrax caribbaeus Rathbun
Mithrox hispidus (Herbst)
Mithrax spinosissimus Lamarck
Mithrax verrucosus H. Milne Edwards
Teleophrys sp. nov.

12

$\begin{array}{llllllll}3 & 4 & 5 & 6 & 7 & 8 & 9 & 10\end{array}$

10

11

Family Pisidae

Chlorilia sp.

Hyas sp.

Rochinia sp.

Family Tychídae

Pitho sp.

Pitho anisodon (von Martins)

Family Dairidae

Daîra sp. nov.

Family Parthenopidae

Mesorhoea sexspinosa Stimpson

Platylambrus sp.

Family Cancridae

Cancer sp.

Family Portunidae

Callinectes jamaicensis Withers

Callinectes sapidus Rathbun

Callinectes toxodes Ordway

$+$

$+$

cf.

+
+
+

$+$

aff.

aff.

aff.

$+$

$+$

$+$

aff.

+

aff.

aff,

Euphylax fortispinosus Collins et al.

Ovalipes sp.

Portunus sp.

portunid gen. et sp. nov.

Family Carcineretidae

Carcineretes woolacotti Withers

Family Carpiliìdae

Carpilius corallinus Herbst

Family Goneplacidae

Nanoplax xanthiformis (A. Mîlne Edwards)

Family Panopeidae

Eurypanopeus sp.

Eurypanopeus abbreviatus (Stimpson)

Eurypanopeus depressus (Smith)

Hexapanopeus caribbaeus (Stimpson)

Lophopanopeus sp. nov. A

Lophopanopeus sp. nov. B

Micropanope nuttingi (Rathbun)

$a$

Micropanope polita Rathbun

Micropanope spinipes A. Milne Edwards

Micropanope sp. nov.

Neopanope sp.

Panopeus herbstii H. Milne Edwards

Panopeus rugosus A. Milne Edwards

Panopeus sp. nov.

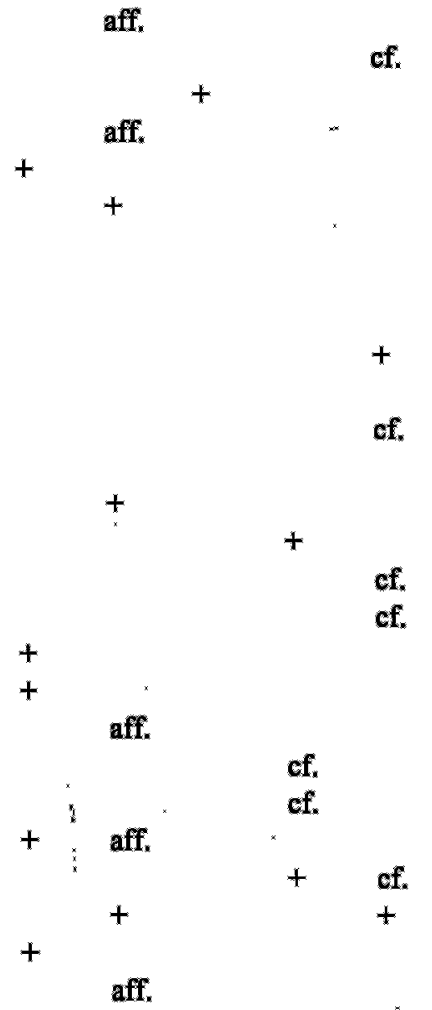


Table 1. Continued.

\begin{tabular}{|c|c|c|c|c|c|c|c|c|c|c|}
\hline Taxon & 2 & 3 & 4 & $\mathbf{5}$ & 6 & 7 & 8 & 9 & 10 & 11 \\
\hline \multicolumn{11}{|l|}{ Family Pilumnidae } \\
\hline Pilumnus pannosus Rathbun & & & & & & aff. & & & & \\
\hline Pilumnus sayi Rathbun & & & & & & & & & cf. & \\
\hline Pilumnus spinossimus Rathbun & & & & & & aff. & & & & \\
\hline \multicolumn{11}{|l|}{ Family Trapeziidae } \\
\hline Trapezia sp. nov. & & & & & + & & & & & \\
\hline \multicolumn{11}{|l|}{ Family Xanthidae } \\
\hline xanthid gen. et sp. nov. & & & & & + & & & & & \\
\hline Chlorodiella sp. nov. & & & & & + & & & & & \\
\hline Eriphia sp. & & & & & & + & & & & \\
\hline Eriphia gonagra xaymacaensis Collins \& D'n & & & & & & & & & + & \\
\hline "Eurypoda" sp. & & & & & & & & & cf. & \\
\hline Eurytium limosum (Say) & & & & & + & aff. & & & & \\
\hline Leptodius sp. nov. & & & & & & & & cf. & & \\
\hline Phymodius maculatus (Stimpson) & & & + & & & & & & & \\
\hline \multicolumn{11}{|l|}{ Xanthilites? rathbunae Withers } \\
\hline Family Ocypodidae & & . & & & & & & & & \\
\hline Uca sp. & & & & & & & & + & & \\
\hline Family Gecarcinidae & & & * & & & & & & & \\
\hline Cardisoma guanhumi Latreille & & & & & & & & & + & + \\
\hline \multicolumn{11}{|l|}{ Family Grapsidae } \\
\hline Pachygrapsus sp. & & & & & & & & + & & \\
\hline Varuna? sp. & & & + & & & & & & & \\
\hline
\end{tabular}

collectors of these specimens, D. Woolacott and C.T. Trechmann. It could be argued that the bias of earlier studies towards the Upper Cretaceous and Eocene has now been overcompensated by our focus on the younger Cenozoic. However, the mid-Cenozoic White Limestone Group (Middle EoceneMiddle Miocene), with its commonly mold-like preservation and low yield of fossils, has deterred many macropaleontologists over the years. The description of abundant carapace material from the Early Miocene Montpelier Formation (Portell \& Collins, in press) must be regarded as a triumph, rather than a failure to find crabs in the rest of the White Limestone Group. New collections of Pliocene and Pleistocene crabs await description by one of us (RWP), particularly from the Late Pliocene Hopegate Formation (dolomitized raised reef) and further taxa from the Falmouth Formation. However, it is anticipated that our program of fieldwork will now shift to the Paleogene, notably the Early-Middle Eocene Chapelton Formation, Yellow Limestone Group, which has already yielded a small diversity of taxa (Table 1). Also high on the agenda is the re-examination of the long-neglected Cretaceous deposits that yielded the material described by Withers.

\section{Acknowledgements}

SKD and RWP gratefully acknowledge the support of National Geographic Society grant \# 7278-02. We thank our many coworkers who have helped us in collecting Jamaican fossil decapods.

\section{References}

Bengtson P. 1988. Open nomenclature. Palaeontology 31: 223-227.

Collins JSH, Donovan SK. 1998. Some new crab records (Crustacea: Decapoda) from the late Pleistocene Port Morant Formation of southeast Jamaica. Bull. Mizunami Fossil Mus. 24 (1997) : 73-77.

Collins JSH, Donovan SK, Dixon HL. 1997. Crabs and barnacles (Crustacea: Decapoda \& Cirripedia) from the late Pleistocene Port Morant Formation of southeast Jamaica, Bull. Mizunami Fossil Mus. 23 (1996): 51-63. 
Collins JSH, Donovan SK, Lindsay W, Simpson GA. 2001. A new species of portunid crab from the early Pleistocene Old Pera beds of Jamaica. Proc. Geol. Ass. 112: 7-12.

Collins JSH, Portell RW. 1998. Decapod, stomatopod and cirripede Crustacea from the Pliocene Bowden shell bed, St. Thomas parish, Jamaica. In: Donovan SK (ed.). The Pliocene Bowden shell bed, southeast Jamaica. Contr. Tert. Quatern. Geol. 35: 113-127.

Donovan SK, Dixon HL. 1998. A fossil land crab from the late Quaternary of Jamaica (Decapoda, Brachyura, Gecarcinidae). Crustaceana 71: 824-826.

Larson DC. 1983. Depositional facies and diagenetic fabrics in the late Pleistocene Falmouth Formation of Jamaica. Norman: University of Oklahoma (unpubl. MS thesis).

Martin JW, Davis GE. 2001. An updated classification of the Recent Crustacea. Nat. Hist. Mus. Los Angeles Co. Sci. ser. 39: 124 pp.

Mitchell SF, Pickerill RK, Stemann TA. 2001. The Port Morant Formation (Upper Pleistocene, Jamaica): hỉgh resolution sedimentology and paleoenvironmental analysis of a mixed carbonate clastic lagoonal succession. Sedim. Geol. 144: 291-306.

Morris SF, 1993. The fossil arthropods of Jamaica. In: Wright RM, Robinson E (eds). Biostratigraphy of Jamaica. Geol. Soc. Amer. Mem. 182: 115-124.

Pickerill RK, Mitchell SF, Donovan SK, Keighley DG. 1998. Sedimentology and palaeoenvironment of the Pliocene
Bowden Formation, southeast Jamaica. In: Donovan SK. (ed.). The Pliocene Bowden shell bed, southeast Jamaica. Contr. Tert. Quatern. Geol. 35: 9-27.

Portell RW, Collins JSH. in press. Decapod crustaceans of the Lower Miocene Montpelier Formation, White Limestone Group of Jamaica. In: Donovan SK. (ed.). The midCainozoic White Limestone Group of Jamaica. Cainozoic Res. 3

Robinson E. 1994. Jamaica. In: Donovan SK, Jackson TA. (eds). Caribbean Geology: An Introduction: 111-127. Kingston: University of the West Indies Publishers" Association.

Underwood CJ, Mitchell SF. in press. Sharks, bony fishes and endodental borings from the Miocene Montpelier Formation (White Limestone Group) of Jamaica. In: Donovan SK (ed.), The mid-Cainozoic White Limestone Group of Jamaica. Cainozoic Res. 3

Withers TH. 1922. On a new brachyurous crustacean from the Upper Cretaceous of Jamaica. Ann. Mag. Nat. Hist. 10: 534-541.

Withers TH. 1924. Some Cretaceous and Tertiary decapod crustaceans from Jamaica. Ann. Mag. Nat. Hist. 13: 8193.

Withers TH. 1927. Ranina trechmanni, a new Cretaceous crab from Jamaica." Geol. Mag. 64: 176-180.

Received: 25 March 2003 